Neuroendocrine tumours of the thymus are rare neoplasms, which account for $2-4 \%$ of all anterior mediastinal tumours. Several endocrinopathies were found to be associated with these tumours, with ectopic ACTH secretion re ported most frequently. Here we provide a description of the syndrome of inappropriate antidiuretic hormone secretion (SIADH) accompanying neuroendocrine thymic carcinoma.

A mediastinal tumour was incidentally found on routine chest $X$-ray examination in a 55-year-old man. After detailed imaging studies, tumour excision was performed. The mediastinal tumour $(11 \times 8 \times 4 \mathrm{~cm})$ presented features of invasion to the left lung and pericardium. Histopathological examination confirmed the diagnosis of thymic neuroendocrine carcinoma. The patient was treated with chemotherapy because of the recurrence. Symptoms of carcinoid syndrome and positive result of the somatostatin receptor scintigraphy justified subsequent introduction of somatostatin analogue therapy. At this point low serum sodium levels accompanied by decreased serum osmolarity and inadequate urine concentration were found. Having excluded other typical causes, SIADH syndrome due to ectopic vasopressin synthesis was assumed. This finding adds to differential diagnosis of hyponatraemia, indicating that SIADH may also accompany thymic neuroendocrine tumours.

Key words: neuroendocrine tumour, thy mus, SIADH, lanreotide.

\section{Lanreotide therapy in a case of thymic neuroendocrine carcinoma associated with syndrome of inappropriate antidiuretic hormone secretion}

\author{
Paweł Gutt, Jakub Fischbach ${ }^{1}$, Daria Baszko-Błaszyk ${ }^{1}$, Marta Fichna ${ }^{1,2}$, \\ Maria Gryczyńska ${ }^{1}$, Marek Ruchała ${ }^{1}$
}

${ }^{1}$ Department of Endocrinology and Metabolism, Poznan University of Medical Sciences, Poznan, Poland

2Institute of Human Genetics, Polish Academy of Sciences, Poznan, Poland

\section{Introduction}

Neuroendocrine tumours of the thymus are very rare neoplasms. They account for only $2-4 \%$ of all neoplasms found in the anterior mediastinum $[1,2]$. A similar percentage is noted in relation to the whole group of neuroendocrine tumours [2]. In the early 1970s, Rosai and Higa were the first to describe neuroendocrine tumours of the thymus and distinguish them from more common thymomas [3]. Worldwide literature provides numerous descriptions of ectopic hormonal activity of these tumours, most commonly presenting with ectopic ACTH secretion which results in Cushing syndrome [4]. However, to our best knowledge, thymic neuroendocrine tumour with concomitant syndrome of inappropriate antidiuretic hormone secretion (SIADH) has not been reported to date.

\section{Case description}

A 55-year-old patient underwent routine chest X-ray screening, which demonstrated an enlarged mediastinal shadow. Subsequent computed tomography (CT) scanning revealed the presence of a hypodense mass $(95 \times 87 \times 70 \mathrm{~mm})$ situated next to the heart, and descending below the aortic arch along the left ventricle (Fig. 1) The mass became enhanced upon contrast medium administration. Positron emission tomography combined with computed tomography imaging (18-F-FDG PET/CT) demonstrated a metabolically active mediastinal tumour with plausible metastases to the local lymph nodes. Left-sided approach thoracotomy was performed and the tumour was removed together with the infiltrated upper lobe of the left lung and a fragment of pericardium. Intraoperative histopathological examination suggested a malignancy of the thymus. The tumour, sized $110 \times 80 \times 40 \mathrm{~mm}$ and presenting focal invasion of the lung through the infiltrated pleura, was deemed to be of neuroendocrine origin. Immunohistochemistry revealed expression of chromogranin $(++)$ and synaptophysin $(+++)$, both characteristic features of neuroendocrine neoplasms. The expression of the Ki67 antigen, considered a marker of mitotic activity, was also assessed; locally it attained $10 \%$ of cells. All above analyses enabled the final diagnosis of neuroendocrine carcinoma derived from the thymus to be established.

Two months later, control imaging studies revealed local neoplastic recurrence with infiltration of the left mediastinal pleura, pericardium, and a new metastatic lesion located paravertabrally, infiltrating the left crus of the elevated diaphragm. Therefore, four courses of chemotherapy, consisting of carboplatin combined with etoposide, were instituted. After these actions the patient was 


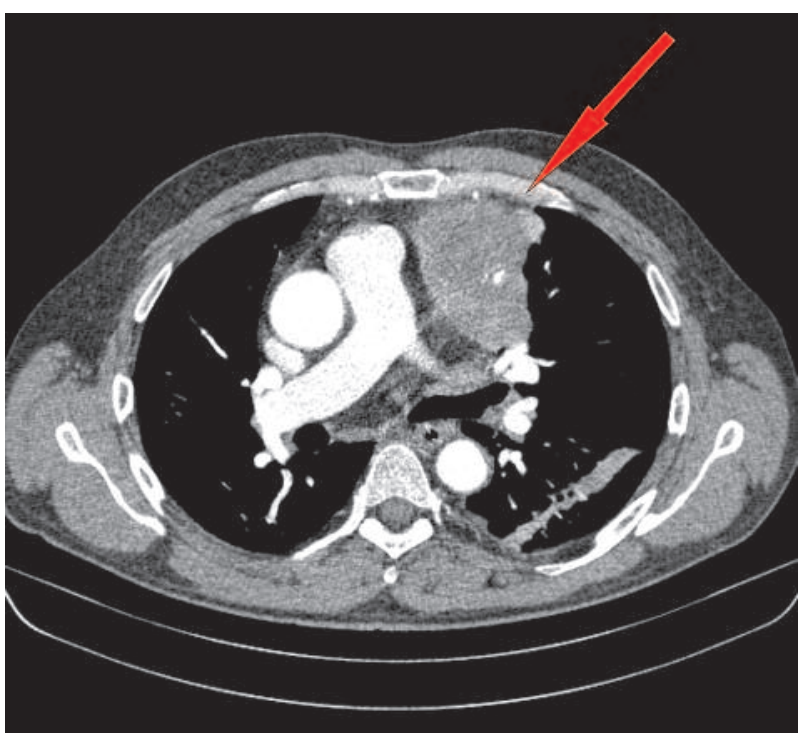

Fig. 1. Computed tomography of the chest. Hypodense mass $(95 \times 87 \times 70 \mathrm{~mm})$ situated next to the heart

sent to the Clinic of Endocrinology to undergo diagnostic tests to qualify for somatostatin analogue therapy. The patient presented typical symptoms of the carcinoid syndrome: facial flushes, blood pressure peaks, abdominal pain and episodes of dyspnoea. General malaise, headaches and episodes of nausea were also observed. Blood pressure and heart rate were within the normal range in several measurements. Biochemical analyses revealed several-fold increase in serum serotonin concentration $(1.75 \mu \mathrm{g} / \mathrm{ml}$, reference: 0.069 0.204 ) and elevated 5 -hydroxyindoleacetic acid (a catabolite of serotonin) excretion in 24-hour urine collections (12.4 mg/24 h, reference: 2-6 mg/24 h), which confirmed hormonal activity of the tumour. Serum levels of a nonspecific marker, chromogranin A, remained within the upper reference range (89 pg/ml, reference: 19-100 pg/ml). Subsequently, a somatostatin analogue-based imaging method was performed: 99mTc-EDDA/HYNIC-TOC (tectrotide) scintigraphy (Fig. 2). It showed enhanced isotope uptake in the left chest, which reflected metastatic change to the chest wall found on repeated chest CT scan. Because of the positive result of the receptor scintigraphy and clinical evidence of tumour hormonal activity, treatment with somatostatin analogue (Lanreotide $120 \mathrm{mg}$ i.m. at 6-week intervals) was considered to be introduced. The patient received a total of 6 lanreotide autogel injections every six weeks. Tolerance of treatment was good. In routine laboratory tests low serum sodium levels were noted (121-123 mmol/l); hence detailed investigation of hyponatraemia was conducted. Low plasma osmolality was observed $\left(225 \mathrm{mOsm} / \mathrm{kg} \mathrm{H}_{2} \mathrm{O}\right)$ with an inadequate degree of the urine concentration (osmolality $387 \mathrm{mOsm} / \mathrm{kg} \mathrm{H}_{2} \mathrm{O}$; $24 \mathrm{~h}$ sodium and potassium excretion 91 and $40 \mathrm{mmol} / \mathrm{l}$, respectively). Trying to exclude potential causes of this abnormality, thyroid function was checked and both hormone concentrations and ultrasound image of the thyroid gland were within the normal range (TSH $1.89 \mathrm{ulU} / \mathrm{ml}$, ref.: 0.27-4.2; fT3 4.79 pmol/l, ref.: 3.95-6.8; fT4 17.75 pmol/l, ref.: 11.5-21.0, normal size and echography). Adrenal cortex insufficiency was excluded (cortisol con-

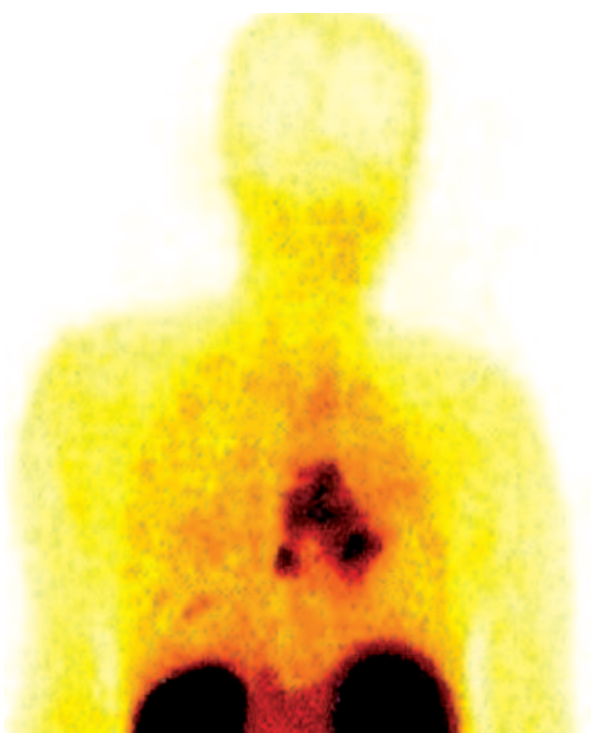

Fig. 2. 99mTc-EDDA/HYNIC (tectrotide) scintigraphy - isotope uptake in the left chest

centration during a day within the normal range e.g.: $472 \mathrm{nmol} / \mathrm{l}$ at 7 a.m., ref.: 171-536, and 203 nmol/l at 6 p.m., ref.: 62-327). Other causes of hyponatraemia, such as heart, kidney or liver failure, were also excluded.

The patient did not follow any particular diet, was not taking any diuretic medications, and his diurnal fluid intake was average, around 2 litres per day. He was diagnosed with syndrome of inappropriate secretion of antidiuretic hormone (SIADH). Fluid restriction to 1 litre per day was prescribed, and oral together with cautious intravenous sodium supplementation was instituted, leading to partial improvement of his natraemia to $129-130 \mathrm{mmol} / \mathrm{l}$. It resulted in partial relief of symptoms due to electrolyte disturbances. Somatostatin analogue treatment proved successful in terms of attenuating symptoms of the carcinoid syndrome; however, due to continuous neoplastic spread, the patient was referred back to the Centre of Oncology in order to provide another chemotherapy protocol (carboplatin and etoposide). Now after the second course of chemotherapy the patient receives lanreotide autogel at a dose of 120 mg every 6 weeks. The situation is stable. Biochemical parameters are normalized. The described tumour size has not increased. Combined chemotherapy and somatostatin analogue treatment is considered to be done in this situation.

\section{Discussion}

SIADH was first reported by Bartter and Schwartz in 1957. Two cases of bronchogenic lung carcinoma with concomitant hyponatraemia and increased natriuresis were presented and their aetiology was linked with plausible excess of antidiuretic hormone (vasopressin, ADH) secretion [5]. The main features of the SIADH comprise:

- hyponatraemia with low plasma osmolality (<280 mmol/kg),

- euvolaemia in clinical examination,

- inappropriately high urine osmolality (> 100 mmol/kg) with regard to low plasma osmolality, and high urinary sodium excretion (> $40 \mathrm{mmol} / \mathrm{l})$, 
- 4. other pathologies which may also associate with hyponatraemia should always be excluded, such as hypothyroidism, adrenal cortex insufficiency, and heart, kidney or liver failure [6].

There are some additional diagnostic criteria of SIADH, although they are rarely applied in everyday routine practice: - water load test: inability to excrete at least $90 \%$ of the water load ( $20 \mathrm{ml} \mathrm{H} \mathrm{H}_{2} \mathrm{O} / \mathrm{kg}$ body mass) within 4 hours after oral administration and/or inability to dilute the urine below $100 \mathrm{mOsm} / \mathrm{kg}$,

- inappropriately high serum ADH levels compared to low plasma osmolarity [7].

The most common causes of SIADH comprise: lung malignancy (small cell lung carcinoma in particular, found in up to $15 \%$ of patients) [8], neoplasms of the gastrointestinal tract, lymphomas, as well as other pulmonary disease: pneumonia, tuberculosis, lung abscess, and central nervous system disorders (tumour, infection, intracranial haemorrhage, brain injury). There are also cases of this syndrome associated with thymic carcinoma [9] and thymoma [10] found in the medical literature. Moreover, SIADH may be an adverse effect of several medications. Antidepressants, especially serotonin reuptake inhibitors, are particularly prone to induce SIADH in as many as $12 \%$ of all hospitalised patients, especially those above 65 years of age [11]. Many other drugs may also lead to inappropriate vasopressin secretion: nonsteroidal anti-inflammatory drugs, quinolones, some sulphonylureas, cyclophosphamide, and even widely used angiotensin convertase inhibitors. Symptomatic hyponatraemia and SIADH were observed after ingestion of 3,4-methylenedioxymethamphetamine (ecstasy) [12]. Rare cases of SIADH were also reported in patients with HIV infection, Guillain-Barré syndrome, sclerosis multiplex, and acute intermittent porphyria [6].

Hyponatraemia, defined as serum sodium concentration below $135 \mathrm{mmol} / \mathrm{l}$, is the most frequent electrolyte disorder encountered among hospitalised patients [13]. It is equally common in outpatient settings. According to the study conducted by Sajadieh et al., the frequency of mild hyponatraemia (130-135 mmol/l) in the population aged 55-75 years, and comprising subjects with no history of cardiovascular disease, stroke or cancer, is around $4 \%$. Moreover, hyponatraemia was found to be an independent risk factor of death and myocardial infarction in this population [14].

Signs and symptoms of hyponatraemia in SIADH correlate with the degree of sodium ion deficit in the extracellular fluid. They range from nearly asymptomatic course in moderate chronic hyponatraemia, through malaise, headaches, nausea and vomiting, vertigo and muscle cramps, to dramatic neurological symptoms with obtundation, breathing problems, seizures, coma and death.

Establishing the aetiology of SIADH is cardinal for efficient treatment; however, not all causes are easy to discern and efficiently eliminate. Symptomatic treatment comprises oral and intravenous sodium chloride supplementation and restriction of fluid intake to $800-1000 \mathrm{ml}$ per day. A new perspective is brought by vaptans, i.e. vasopressin receptor antagonists. They inhibit the activation of the $\mathrm{V} 2$ receptors in kidney distal convoluted tubules and collecting ducts, leading to increased excretion of free water with unaffected urine excretion of ions, which in turn results in an increase of serum sodium level. None of the recently used methods displayed this kind of causative mechanism of action [15].

Neuroendocrine tumours of the thymus are very rare. Their incidence is estimated at approximately 1-2 cases per $10,000,000$ inhabitants per year [16]. Peak incidence is at the sixth decade of life, with an excess of males (male to female ratio $=3: 1$ ) [17]. Neuroendocrine tumours account for only $5 \%$ of all thymic neoplasms [18]. Symptoms are usually nonspecific and related to the mass effect: dyspnoea, cough, and superior vena cava syndrome. Some cases remain asymptomatic. Diagnostic procedures commonly comprise classical imaging studies: chest X-ray, computed tomography scans, magnetic resonance imaging. The histopathological confirmation of the neuroendocrine character of the tumour provides the rationale for receptor scintigraphy with radiolabelled somatostatin analogues. The technique relies on the presence of transmembrane somatostatin receptors on the surface of the tumour cells [19]. This precious tool enables us to visualise potential metastatic changes, and may also provide an indication for systemic treatment with somatostatin analogues. By means of the activation of type 2 and type 5 somatostatin receptors, these pharmacological agents inhibit cell proliferation and angiogenesis, decrease hormonal activity of the tumour, and also may induce apoptosis [20].

Radiolabelled somatostatin analogues which emit beta radiation, i.e. [90Y-DOTA ${ }^{0}$, Tyr $^{3}$ ]-octreotide or ${ }^{177}$ Lu-(DOTA0,Tyr $\left.{ }^{3}\right)$ octreotate, are another therapeutic option for neuroendocrine tumours. Poorly differentiated tumours with a high proliferation index require chemotherapy, combining different cytostatic drugs. Clinical trials for several new, recently developed agents, such as blockers of angiogenesis (bevacizumab), receptor tyrosine kinase inhibitors (sunitinib), and rapamycin derivative (everolimus), are still underway [21]. Survival rates mainly depend on the degree of histological maturity, disease stage at diagnosis, and operability of the tumour. Some reports suggest that coexisting endocrinopathy significantly deteriorates the prognosis of 5 -year survival compared to patients without hormonal disorders (35\% vs. 65\%) [4].

In summary, hyponatraemia is the most common electrolyte disorder found in hospitalized patients, with SIADH being the most frequent reason for this condition [6]. Diagnostic procedures may appear troublesome due to the vast number of potential aetiologies, which require careful exclusion. The case presented here indicates another possible cause of hyponatraemia, which should also be considered.

\section{References}

1. Moran CA, Suster S. Spindle-cell neuroendocrine carcinoma of the thymus: a clinicopathologic and immunohistochemical study of seven cases. Mod Pathol 1999; 12: 587-91.

2. Jensen RT, Norton JA,. Carcinoid tumors and carcinoid syndrome. Cancer: Principles and practice of oncology. 5th edition. DeVita VT, Hellman S, Rosenberg SA (eds.). J.B Lippincott Company, Philadelphia 1997: 1704-23.

3. Rosai J, Higa E. Mediastinal endocrine neoplasm, of probable thymic origin, related to carcinoid tumor: clinicopathologic study of 8 cases. Cancer 1972; 29: 1061-74.

4. Ruffini E, Oliaro A, Novero D, Campisi P, Filosso PL. Neuroendocrine tumors of the thymus. Thorac Surg Clin 2011; 21: 13-23. 
5. Schwartz WB, Bennet W, Curelop S, Bartter FC. A syndrome of renal sodium loss and hyponatremia probably resulting from inappropriate secretion of antidiuretic hormone. Am J Med 1957; 23: 529-42.

6. Hannon MJ, Thomson CJ. The syndrome of inappropriate antidiuretic hormone: prevalence, causes and consequences. Eur J Endocrinol 2010; 162: S5-S12.

7. Smith MD, McKenna K, Thomson C. Hyponatremia. Clinical Endocrinology 2000; 52; 667-8.

8. Sorensen JB, Andersen MK, Hansen HH. Syndrome of innapropriate secretion of antidiuretic hormone (SIADH) in malignant disease. J Intern Med 1995; 238: 97-110.

9. Walid MS, Troup EC, Robinson JS Jr. Brain metastasis from thymic carcinoma in association with SIADH and pituitary enlargement: a case report. South Med J. 2008; 101: 764-6.

10. Galesic K, Krizanac S, Vrkljan M, Ljubanovic D. Syndrome of inappropriate secretion of antidiuretic hormone due to malignant thymoma. Nephron 2002; 91: 752-4.

11. Bouman WP, Pinner G, Johnson H. Incidence of selective serotonin reuptake inhibitor induced hyponatremia due to syndrome of inappropriate antidiuretic hormone secretion in the elderly. Int J Geriatr Psychiatry 1998; 13: 113-5.

12. Holden R, Jackson MA. Near-fatal hyponatremic coma due to vasopressin over-secretion after “ecstasy' (3,4-MDMA). Lancet 1996; 347: 105

13. Baran D, Hutchison TA. Outcome of hyponatraemia in general hospital population. Clinical Nephrology 1984; 22: 72-6.

14. Sajadieh A, Binici Z, Mouridsen MR, Nielsen OW, Hansen JF, Haugaard SB. Mild hyponatremia carries a poor prognosis in community subject. American Journal of Medicine 2009; 122: 679-86.

15. Olszewski W, Głuszek J. Vasopressin antagonists in treatment of hyponatremia. Pol Arch Med Wewn 2007; 117: 356-62.

16. Oberg K, Jelic S. Neuroendocrine bronchial and thymic tumors: ESMO clinical recommendation for diagnosis, treatment and follow-up. Ann Oncol 2008; 19: 102-3.

17. Dusmet ME, McKneally MF. Pulmonary and thymic carcinoid tumors. World J Surg 1996; 20: 189-95.

18. Shimosato Y, Mukai K. Tumors of the madiastinum. 3rd ed. Washington, DC: Armed Forces Institute of Pathology 1995.

19. Kaltas G, Rockall A, Papadogias D, Reznek R, Grossman AB. Recent advances in radiological and radionuclide imaging and therapy of endocrine tumors. Eur J Endocrinol 2004; 151: 15-27.

20. Pawlikowki M. Receptory somatostatynowe w guzach neuroendokrynnych. Guzy neuroendokrynne układu pokarmowego. Kos-Kudła B (ed.). Via medica, Gdańsk 2010; 25-32.

21. Phan AT, Oberg K, Choi J, Harrison LH, Hassan MM, Strosberg JR, Krenning EP, Kocha W, Woltering EA, Maples WJ. NANETS Consensus guideline for diagnosis and management of neuroendocrine tumors. Welldifferentiated neuroendocrine Tumors of the thorax (Includes lung and thymus). Pancreas 2010; 39: 784-98.

\section{Address for correspondence}

Paweł Gut MD, PhD

Department of Endocrinology and Metabolism

Poznan University of Medical Sciences

Przybyszewskiego 49

60-355 Poznan

tel. 607392922

e-mail: gutpj@poczta.onet.pl 